\title{
Ketamine attenuates the lipopolysaccharide-induced inflammatory response in cultured $\mathrm{N} 2 \mathrm{a}$ cells
}

\author{
CHUN YANG $^{1}$, RI-YUE JIANG ${ }^{2}$, JIANG SHEN ${ }^{1}$, TAO HONG $^{1}$, NING LIU ${ }^{3}$, \\ LIANG-CAI DING ${ }^{3}$, DA-MING WANG ${ }^{3}$, LU-JUN CHEN ${ }^{4}$, BIN XU $^{5}$ and BIN ZHU ${ }^{3}$ \\ Departments of ${ }^{1}$ Anesthesiology, ${ }^{2}$ Radiotherapy and ${ }^{3}$ Critical Care Medicine; ${ }^{4}$ Comprehensive Laboratory; \\ ${ }^{5}$ Department of Tumor Biological Treatment, The Third Affiliated Hospital of \\ Soochow University, Changzhou, Jiangsu 213003, P.R. China
}

Received January 20, 2013; Accepted April 26, 2013

DOI: $10.3892 / \mathrm{mmr} .2013 .1465$

\begin{abstract}
The use of ketamine is recommended in patients with sepsis undergoing surgery due to its anti-inflammatory effects. However, a paucity of data exists with regard to the anti-inflammatory effects of ketamine in the central nervous system. Therefore, the present study aimed to investigate the effect of ketamine on lipopolysaccharide (LPS)-induced inflammatory responses in cultured Neuro2a (N2a) cells and to elucidate its potential mechanism of action. N2a cells were randomly divided into the following 3 groups $(n=6)$ : The DMEM culture solution administration alone group, the $0.5 \mu \mathrm{mol} / 1 \mathrm{LPS}$ administration alone group and the $1 \mu \mathrm{mol} / 1$ ketamine plus $0.5 \mu \mathrm{mol} / 1$ LPS administration group. The expression levels of interleukin (IL)-1 $\beta$, IL-6, tumor necrosis factor (TNF)- $\alpha$, nuclear factor $(\mathrm{NF})-\kappa \mathrm{B}$ and inducible nitric oxide synthase (iNOS) were determined. LPS-treated N2a cells exhibited a significant increase in the expression levels of IL-1 $\beta$, IL-6, TNF- $\alpha, N F-\kappa B$ and iNOS, while the administration of ketamine eliminated the LPS-induced production of IL-1 $\beta$, IL-6, TNF- $\alpha$, NF- $\kappa$ B and iNOS. Based on our data, we hypothesized that the anti-inflammatory effect exerted by ketamine on $\mathrm{N} 2 \mathrm{a}$ cells was potentially due to the inhibition of $\mathrm{NF}-\kappa \mathrm{B}$ and iNOS.
\end{abstract}

\section{Introduction}

Lipopolysaccharide (LPS), a component of the Gram-negative bacterial cell wall, is a potent inducer of inflammation (1). The inflammatory response to Gram-negative bacterial infections in the central nervous system remains a major cause of

Correspondence to: Dr Bin Zhu, Department of Critical Care Medicine, The Third Affiliated Hospital of Soochow University, No. 185 Juqian Street, Changzhou, Jiangsu 213003, P.R. China E-mail: anesthesia3143@163.com

Key words: ketamine, lipopolysaccharide, pro-inflammatory cytokines, nuclear factor- $\kappa \mathrm{B}$, inducible nitric oxide synthase, Neuro2a cells neurological disease (2). Several studies have suggested that neuroinflammation is implicated in the pathology of several neurodegenerative diseases, including Alzheimer's disease, Parkinson's disease and multiple sclerosis (3-5). It has been demonstrated that elevated levels of pro-inflammatory cytokines are capable of inducing the onset of a number of neurodegenerative diseases (6). Although the pathologies of different neurodegenerative diseases are distinct, neuroinflammation is commonly involved (7). It has been suggested that the inhibition of neuroinflammation prevents the onset and reduces the symptoms of neurodegenerative diseases $(6,7)$. A significant body of evidence has observed LPS-induced neuroinflammation, characterized by the elevated expression levels of interleukin (IL)-1 $\beta$, IL-6 and tumor necrosis factor (TNF)- $\alpha$, in the central nervous system of animal models with neurological diseases (8-10).

Ketamine, a commonly used anesthetic agent, is recommended for use in patients with sepsis (11). Clinically, the majority of anesthesiologists have observed that ketamine is capable of increasing blood pressure, particularly in patients with sepsis. Collectively, several studies have demonstrated that ketamine is able to maintain circulatory stability, mainly as a result of inhibiting the secretion of pro-inflammatory cytokines (12-14). Koga et al (15) have demonstrated that ketamine is capable of decreasing the mortality rate in rats with endotoxin-induced shock, as well as the activity of TNF- $\alpha$. Another study by Taniguchi et al (16) demonstrated that ketamine is able to inhibit the release of IL-1 $\beta$ and IL-6. Furthermore, it has been shown that ketamine inhibits the LPS-induced inflammatory response in a dose-dependent manner (17).

However, a paucity of data exists with regard to the anti-inflammatory effects of ketamine in the central nervous system. Therefore, in the present study, we aimed to investigate the effect of ketamine on the LPS-induced inflammatory response in cultured Neuro2a (N2a) cells and to elucidate its potential mechanism of action.

\section{Materials and methods}

Reagents. Ketamine was purchased from Fujian Gutian Pharmaceutical Company (Fujian, China). IL-1 $\beta$, IL-6 and 
TNF- $\alpha$ ELISA kits were purchased from Nanjing Jiancheng Bioengineering Company (Nanjing, China). Primary antibodies against nuclear factor (NF)- $\mathrm{kB}$ and inducible nitric oxide synthase (iNOS) were purchased from Cell Signaling Technology, Inc. (Danvers, MA, USA). The study was approved by the ethics committee of Soochow University, Suzhou, China.

Cell culture. N2a cells were obtained from Medical College, Soochow University, Suzhou, China. The mouse N2a neuroblastoma cell culture was performed as described previously $(18,19)$. N2a cells were maintained in DMEM (Gibco Co., New York, USA) culture solution supplemented with $10 \%$ FBS (Gibco), $0.3 \mathrm{mM} \mathrm{L}$-glutamine and $50 \mathrm{U} / \mathrm{ml}$ penicillin/streptomycin. N2a cells with $70 \%$ confluence were randomly divided into 3 groups (6 duplicates), and each culture well contained $2 \times 10^{5} \mathrm{~N} 2 \mathrm{a}$ cells. DMEM solution, $0.5 \mu \mathrm{mol} / 1 \mathrm{LPS}$ or $1 \mu \mathrm{mol} / 1$ ketamine plus $0.5 \mu \mathrm{mol} / 1$ LPS (Sigma, Oakville, ON, Canada) were randomly administered into 18 culture wells (n=6/group). Forty-eight hours after incubation, N2a cells were prepared for analysis.

ELISA. The concentration of cytokines was determined using ELISA kits. Forty-eight-well microtiter plates were coated with a monoclonal antibody to mouse cytokines and were incubated in hydrogen bicarbonate buffer ( $\mathrm{pH}$ 9.6) overnight at $4^{\circ} \mathrm{C}$. In order to avoid non-specific binding, the wells were blocked with $0.5 \%$ bovine serum albumin (BSA, Equitech-Bio Inc., Kerrville, TX, USA) in phosphate-buffered saline (PBS, Sigma; $60 \mathrm{mmol} / \mathrm{l}, \mathrm{pH}$ 7.4) for $1 \mathrm{~h}$ at room temperature. Undiluted culture supernatants were added in duplicates and incubated for $2 \mathrm{~h}$ at $37^{\circ} \mathrm{C}$. Antibodies to human cytokines coupled with biotin (Pharmingen, San Diego, CA, USA) were used as secondary antibodies $(1.25 \mu \mathrm{g} / \mathrm{ml})$ and were incubated with the plates at $37^{\circ} \mathrm{C}$ for $2 \mathrm{~h}$. Following incubation, avidin-peroxidase $(2.5 \mu \mathrm{g} / \mathrm{ml})$ was added for $1 \mathrm{~h}$ and then $0.5 \mathrm{mg} / \mathrm{ml}$ orthophenylenediamine dissolved in citrate buffer (pH 5.0) with $0.01 \% \mathrm{H}_{2} \mathrm{O}_{2}$ was used as a substrate solution. After stopping the enzyme reaction with $25 \%$ sulphuric acid, optical density was measured in a microtiter plate reader at $450 \mathrm{~nm}$. Recombinant cytokines were used to establish a standard curve to which the results obtained with the culture supernatants were correlated.

Western blotting. The N2a cells were washed with PBS and lysed in 1.3X SDS-containing sample buffer without 1,4-dithio-dl-threitol (DTT) or bromophenol blue containing $100 \mu \mathrm{M}$ orthovanadate. Protein levels were determined using the bicinchoninic acid (BCA) method, according to the manufacturer's instructions. BSA was used as a standard. Prior to electrophoresis, a mixture of bromophenol blue and DTT (final concentration, $10 \mathrm{mM}$ ) was added to the samples. For western blotting, $50 \mu \mathrm{g}$ of the total protein from each sample was separated by SDS-PAGE under reducing conditions. The proteins were then transferred onto polyvinylidene fluoride (PVDF) membranes (Nanjing Jiancheng Bioengineering Company, Nanjing, China). The membranes were blocked for $2 \mathrm{~h}$ at room temperature using non-fat dried milk blotting grade blocker and incubated overnight with primary antibodies. The primary antibodies used were goat anti-NF- $\mathrm{KB}(1: 2,000)$ and rabbit anti-iNOS $(1: 1,000)$. Primary antibodies were diluted in Tris-+6buffered saline (TBS, Thermo Fisher Scientific Inc., Rockford, IL, USA) containing $0.1 \%$ Tween-20 (TBS-T) and $1 \%$ BSA. Following extensive washing (three times for $15 \mathrm{~min}$ each in TBS-T), the NF- $\kappa$ B and iNOS protein levels were measured with horseradish peroxidase-conjugated rabbit antigoat $\operatorname{IgG}(1: 100,000$ dilution) using chemiluminescence (ECL) reagents (Beyotime Company, Nantong, China). Equal protein loading and transfer were assessed by the subjection of each sample to western blotting for GAPDH (rabbit anti-GAPDH IgG, 1:5,000 dilution).

Statistical analysis. Data are expressed as the mean \pm SD. Statistical analyses were performed using one-way ANOVA and post hoc analyses were performed using the least significant difference test. These statistical analyses were conducted using SPSS version 17.0 (IBM, Chicago, IL, USA). P $<0.05$ was considered to indicate a statistically significant result.

\section{Results}

LPS administration increases the expression levels of IL-1 $\beta$, $I L-6$ and TNF- $\alpha$ in cultured N2a cells. The administration of LPS alone significantly increased the expression levels of IL-1 $\beta$, IL- 6 and TNF- $\alpha$ compared with DMEM administration alone in cultured N2a cells $(\mathrm{P}<0.05$; Figs. 1-3).

Ketamine attenuates the LPS-induced upregulation of IL-1 $\beta$, IL- 6 and TNF- $\alpha$ in cultured N2a cells. Compared with the LPS administration alone group, the administration of LPS plus ketamine group demonstrated a significant decrease in the levels of IL-1 $\beta$, IL- 6 and TNF- $\alpha$ in cultured N2a cells $(\mathrm{P}<0.05$; Figs. 1-3).

Effects of ketamine and/or LPS on the expression levels of $N F-\kappa B$ and iNOS in cultured N2a cells. Western blotting was used to assess the expression levels of NF- $\mathrm{KB}$ and iNOS, and the results demonstrated that the administration of LPS alone significantly increased the expression levels of NF- $\mathrm{KB}$ and iNOS compared with DMEM administration alone in cultured N2a cells. By contrast, the administration of ketamine attenuated the LPS-induced upregulation of NF- $\mathrm{kB}$ and iNOS $(\mathrm{P}<0.05$; Figs. 4 and 5).

\section{Discussion}

In the present study, several inflammatory cytokines, including IL-1 $\beta$, IL-6 and TNF- $\alpha$, were upregulated in LPS-treated N2a cells, while the administration of ketamine attenuated the LPS-induced inflammatory response. In addition, we demonstrated that the expression levels of NF- $\mathrm{kB}$ and iNOS were increased following LPS treatment, while they were downregulated following ketamine treatment.

Several lines of evidence have indicated that ketamine, an $\mathrm{N}$-methyl-D-aspartic acid (NMDA) receptor antagonist, has a significant positive effect on the regulation of the inflammatory response $(13,14)$. Several studies have reported that ketamine exhibits an inhibitory effect on the inflammatory response in vitro (20-22). In addition, Shibakawa et al (23) demonstrated that ketamine significantly inhibits the LPS-induced inflam- 


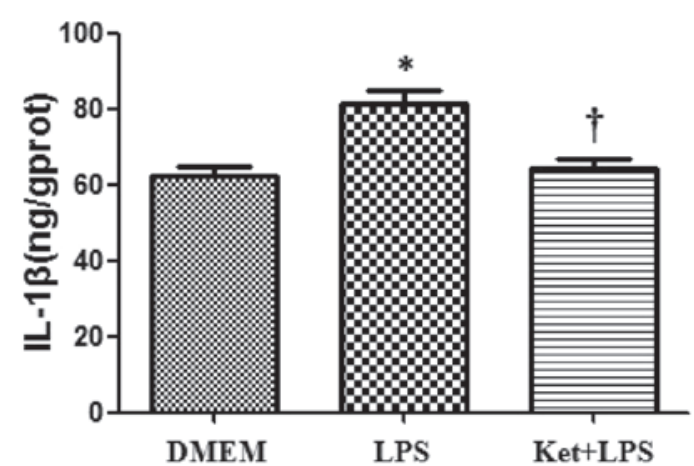

Figure 1. Expression of IL-1 $\beta$ following LPS and/or Ket treatment in cultured N2a cells. "P $<0.05$ compared with DMEM administration alone; ${ }^{\dagger} \mathrm{P}<0.05$ compared with LPS administration. IL, interleukin; LPS, lipopolysaccharide; Ket, ketamine; N2a, Neuro2a.

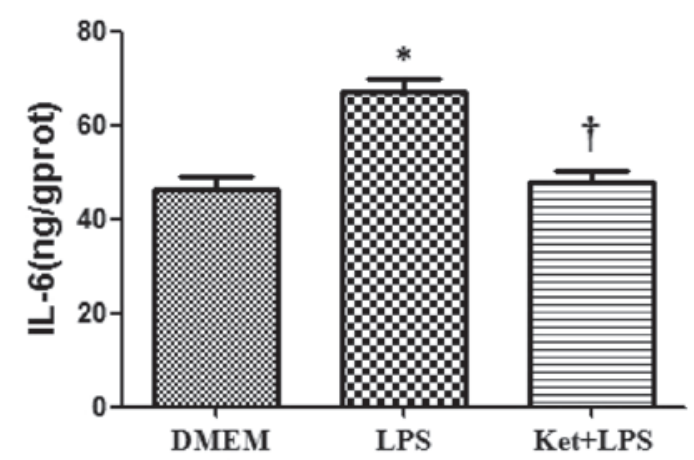

Figure 2. Expression of IL-6 following LPS and/or Ket treatment in cultured N2a cells. "P<0.05 compared with DMEM administration alone; ${ }^{\dagger} \mathrm{P}<0.05$ compared with LPS administration. IL, interleukin; LPS, lipopolysaccharide; Ket, ketamine; N2a, Neuro2a.

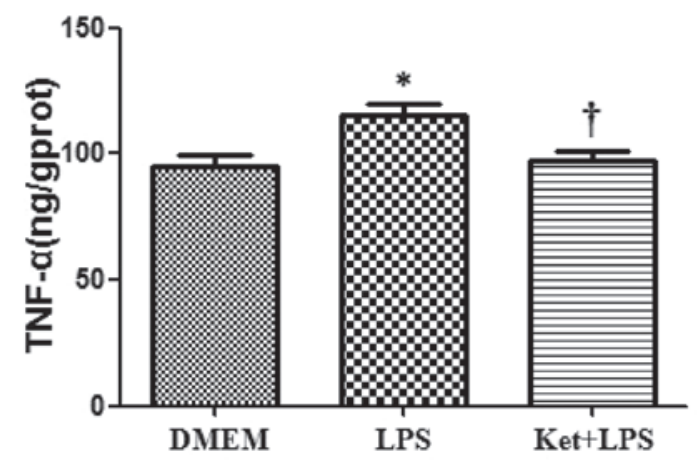

Figure 3. Expression of TNF- $\alpha$ following LPS and/or Ket treatment in cultured N2a cells. ${ }^{*} \mathrm{P}<0.05$ compared with DMEM administration alone; ${ }^{\dagger} \mathrm{P}<0.05$ compared with LPS administration. TNF- $\alpha$, tumor necrosis factor- $\alpha$; LPS, lipopolysaccharide; Ket, ketamine; N2a, Neuro2a.

matory response in microglial cells. However, in the present study, we used N2a cells to investigate the anti-inflammatory effect of ketamine. Our results demonstrated that ketamine exerted an inhibitory effect on the LPS-stimulated inflammatory response in $\mathrm{N} 2 \mathrm{a}$ cells.

$\mathrm{NF}-\mathrm{kB}$ has been shown to be important in regulating the expression of a number of genes involved in cell survival,

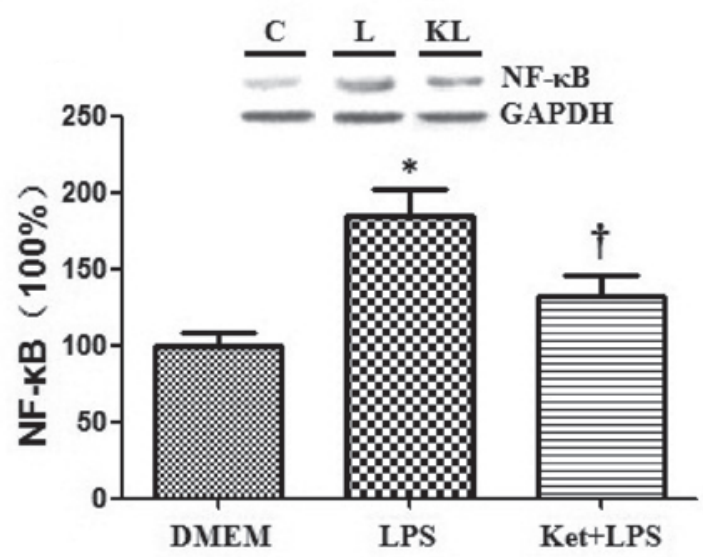

Figure 4. Expression of NF- $\mathrm{kB}$ following LPS and/or ketamine treatment in cultured N2a cells. " $\mathrm{P}<0.05$ compared with DMEM administration alone; ${ }^{\dagger} \mathrm{P}<0.05$ compared with LPS administration. NF- $\kappa \mathrm{B}$, nuclear factor- $\kappa \mathrm{B}$; LPS, lipopolysaccharide; Ket, ketamine; N2a, Neuro2a; C, control group; L, lipopolysaccharide group; KL, ketamine + lipopolysaccharide group.

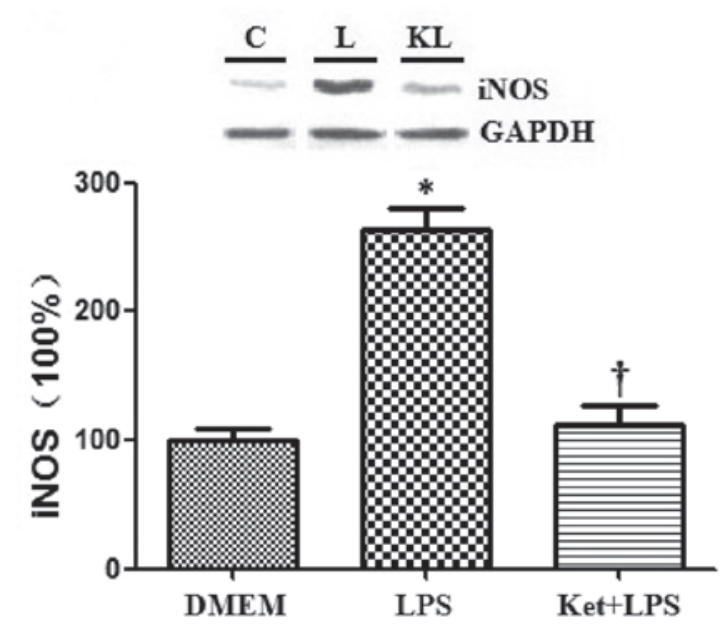

Figure 5. Expression of iNOS following LPS and/or ketamine treatment in cultured N2a cells. " $\mathrm{P}<0.05$ compared with DMEM administration alone; ${ }^{\dagger} \mathrm{P}<0.05$ compared with LPS administration. iNOS, inducible nitric oxide synthase; LPS, lipopolysaccharide; Ket, ketamine; N2a, Neuro2a; C, control group; L, lipopolysaccharide group; KL, ketamine + lipopolysaccharide group.

inflammatory processes and neuronal degeneration (24). Dysregulation of the NF- $\kappa \mathrm{B}$ signaling pathway may result in chronic neuroinflammation and neuronal death, thus the NF- $\mathrm{KB}$ signaling pathway is important in the regulation of neuronal function $(25,26)$. A recent study by Wu et al $(27)$ demonstrated that the inhibitory effect of ketamine on the LPS-induced inflammatory response may be mediated by the suppression of NF-кB activation. In addition, Yu et al (28) revealed that ketamine is able to inhibit pulmonary NF- $\mathrm{KB}$ activity during endotoxemia in rats. The results of the present study were consistent with previous findings demonstrating that LPS significantly activates the expression of NF- $\mathrm{KB}$, while ketamine is capable of inhibiting the LPS-induced activation of NF- $\mathrm{KB}$.

In the present study, the upregulation of NF- $\mathrm{B}$ was observed in LPS-treated N2a cells. It has been reported that NF- $\mathrm{KB}$ activation upregulates the production of iNOS, which 
leads to the upregulation of nitric oxide production during the inflammatory response (29). NF- $\kappa \mathrm{B}$-dependent activation of iNOS promotes the inflammatory response (30). Therefore, we hypothesized that $\mathrm{NF}-\kappa \mathrm{B}$ and iNOS are important in mediating the inhibitory effect of ketamine on the inflammatory response in the central nervous system. Our results demonstrated that ketamine was capable of inhibiting the LPS-induced activation of iNOS, which confirmed our hypothesis.

Several studies have reported that the pathogenesis of numerous types of neurodegenerative diseases, including Alzheimer's disease, Parkinson's disease and multiple sclerosis, are likely to be associated with increased levels of pro-inflammatory cytokines in the central nervous system (3-5). Notably, as a widely used anesthetic agent, ketamine has attracted increasing attention for the treatment of refractory depression. To the best of our knowledge, no other study has reported the therapeutic effects of ketamine in other inflammation-associated neurological and/or psychiatric diseases to date. Large-scale studies are required to fully elucidate the therapeutic effects of ketamine, thereby expanding its clinical application.

In conclusion, ketamine is capable of exerting an inhibitory effect on the LPS-induced inflammatory response in cultured $\mathrm{N} 2 \mathrm{a}$ cells, and its mechanism is likely to be associated with the inhibition of NF- $\mathrm{KB}$ and iNOS.

\section{References}

1. Tuo W, Ott TL, Liu S and Bazer FW: Intrauterine infusion of bacterial lipopolysaccharide (LPS) prior to mating has no adverse effect on fertility, fetal survival and fetal development. J Reprod Immunol 42: 31-39, 1999.

2. Glass CK, Saijo K, Winner B, Marchetto MC and Gage FH: Mechanisms underlying inflammation in neurodegeneration. Cell 140: 918-934, 2010.

3. Dhib-JalbutS,ArnoldDL,ClevelandDW,etal: Neurodegeneration and neuroprotection in multiple sclerosis and other neurodegenerative diseases. J Neuroimmunol 176: 198-215, 2006.

4. Rozemuller AJ, Jansen C, Carrano A, et al: Neuroinflammation and common mechanism in Alzheimer's disease and prion amyloidosis: amyloid-associated proteins, neuroinflammation and neurofibrillary degeneration. Neurodegener Dis 10: 301-304, 2012.

5. Cooper-Knock J, Kirby J, Ferraiuolo L, Heath PR, Rattray M and Shaw PJ: Gene expression profiling in human neurodegenerative disease. Nat Rev Neurol 8: 518-530, 2012.

6. Weiner HL and Selkoe DJ: Inflammation and therapeutic vaccination in CNS diseases. Nature 420: 879-884, 2002.

7. Minghetti L: Role of inflammation in neurodegenerative diseases. Curr Opin Neurol 18: 315-321, 2005.

8. Ambrosini A, Louin G, Croci N, Plotkine $M$ and Jafarian-Tehrani M: Characterization of a rat model to study acute neuroinflammation on histopathological, biochemical and functional outcomes. J Neurosci Methods 144: 183-191, 2005.

9. Burd I, Bentz AI, Chai J, et al: Inflammation-induced preterm birth alters neuronal morphology in the mouse fetal brain. J Neurosci Res 88: 1872-1881, 2010.

10. Belarbi K, Jopson T, Tweedie D, et al: TNF- $\alpha$ protein synthesis inhibitor restores neuronal function and reverses cognitive deficits induced by chronic neuroinflammation. J Neuroinflammation 9: 23, 2012.
11. Park GR, Manara AR, Mendel L and Bateman PE: Ketamine infusion. Its use as a sedative, inotrope and bronchodilator in a critically ill patient. Anaesthesia 42: 980-983, 1987.

12. Cho JE, Shim JK, Choi YS, Kim DH, Hong SW and Kwak YL: Effect of low-dose ketamine on inflammatory response in off-pump coronary artery bypass graft surgery. Br J Anaesth 102: 23-28, 2009.

13. Tsao CM, Wu CC, Wang JJ, Wong CS, Tsai SK and Ho ST: Intravenous anesthetics in sepsis. Acta Anaesthesiol Taiwan 43: $153-163,2005$.

14. Taniguchi T and Yamamoto K: Anti-inflammatory effects of intravenous anesthetics on endotoxemia. Mini Rev Med Chem 5: 241-245, 2005.

15. Koga K, Ogata M, Takenaka I, Matsumoto T and Shigematsu A: Ketamine suppresses tumor necrosis factor-alpha activity and mortality in carrageenan-sensitized endotoxin shock model. Circ Shock 44: 160-168, 1994.

16. Taniguchi T, Kanakura H, Takemoto $\mathrm{Y}$, Kidani $\mathrm{Y}$ and Yamamoto K: Effects of ketamine and propofol on the ratio of interleukin-6 to interleukin-10 during endotoxemia in rats. Tohoku J Exp Med 200: 85-92, 2003.

17. Taniguchi T, Takemoto Y, Kanakura H, Kidani $\mathrm{Y}$ and Yamamoto K: The dose-related effects of ketamine on mortality and cytokine responses to endotoxin-induced shock in rats. Anesth Analg 97: 1769-1772, 2003.

18. Strenge K, Schauer R and Kelm S: Binding partners for the myelin-associated glycoprotein of N2A neuroblastoma cells. FEBS Lett 444: 59-64, 1999

19. Björkdahl C, Sjögren MJ, Winblad B and Pei JJ: Zinc induces neurofilament phosphorylation independent of p70 S6 kinase in N2a cells. Neuroreport 16: 591-595, 2005.

20. Kawasaki T, Ogata M, Kawasaki C, Ogata J, Inoue Y and Shigematsu A: Ketamine suppresses proinflammatory cytokine production in human whole blood in vitro. Anesth Analg 89: 665-669, 1999 .

21. Takenaka I, Ogata M, Koga K, Matsumoto T and Shigematsu A: Ketamine suppresses endotoxin-induced tumor necrosis factor alpha production in mice. Anesthesiology 80: 402-408, 1994.

22. Shimaoka M, Iida T, Ohara A, et al: Ketamine inhibits nitric oxide production in mouse-activated macrophage-like cells. $\mathrm{Br}$ J Anaesth 77: 238-242, 1996.

23. Shibakawa YS, Sasaki Y, Goshima Y, et al: Effects of ketamine and propofol on inflammatory responses of primary glial cell cultures stimulated with lipopolysaccharide. Br J Anaesth 95: 803-810, 2005.

24. Jones E, Adcock IM, Ahmed BY and Punchard NA: Modulation of LPS stimulated NF-kappaB mediated Nitric Oxide production by PKCepsilon and JAK2 in RAW macrophages. J Inflamm (Lond) 4: 23, 2007.

25. Bonini SA, Ferrari-Toninelli G, Uberti D, et al: Nuclear factor $\kappa \mathrm{B}$-dependent neurite remodeling is mediated by Notch pathway. J Neurosci 31: 11697-11705, 2011.

26. Makarov SS: NF-kappaB as a therapeutic target in chronic inflammation: recent advances. Mol Med Today 6: 441-448, 2000.

27. Wu Y, Li W, Zhou C, et al: Ketamine inhibits lipopolysaccharide-induced astrocytes activation by suppressing TLR4/NF-KB pathway. Cell Physiol Biochem 30: 609-617, 2012.

28. Yu M, Shao D, Feng X, Duan M and Xu J: Effects of ketamine on pulmonary TLR4 expression and NF-kappa-B activation during endotoxemia in rats. Methods Find Exp Clin Pharmacol 29: 395-399, 2007.

29. Baldwin AS Jr: The NF-kappa B and I kappa B proteins: new discoveries and insights. Annu Rev Immunol 14: 649-683, 1996.

30. Han S, Lee JH, Kim C, et al: Capillarisin inhibits iNOS, COX-2 expression, and proinflammatory cytokines in LPS-induced RAW 264.7 macrophages via the suppression of ERK, JNK, and $\mathrm{NF}-\kappa \mathrm{B}$ activation. Immunopharmacol Immunotoxicol 35: 34-42, 2013. 